\title{
Intentional and automatic measures of specific-category effect in the semantic impairment of patients with Alzheimer's disease
}

\author{
Roberta Perri ${ }^{\mathrm{a}, *}$, Giovanni A. Carlesimo ${ }^{\mathrm{a}, \mathrm{b}}$, Gian Daniele Zannino ${ }^{\mathrm{a}}$, Marco Mauri ${ }^{\mathrm{c}}$, \\ Barbara Muolo ${ }^{\mathrm{d}}$, Carla Pettenati ${ }^{\mathrm{d}}$, Carlo Caltagirone ${ }^{\mathrm{a}, \mathrm{b}}$ \\ a IRCCS Fondazione Santa Lucia, V. Ardeatina, 306, 00179 Rome, Italy \\ ${ }^{\mathrm{b}}$ Clinica Neurologica, Università di Roma Tor Vergata, Rome, Italy \\ ${ }^{c}$ Clinica Neurologica, Università dell'Insubria di Varese, Varese, Italy \\ ${ }^{d}$ Centro Regionale Alzheimer, UOC Neurologia, Ospedale Rho-Passirana, Rho, Italy
}

Received 5 July 2002; received in revised form 7 March 2003; accepted 7 March 2003

\begin{abstract}
The breakdown of semantic knowledge relative to living and non-living categories was studied in patients with Alzheimer's disease (AD). The same living and non-living items were used in a semantic battery and in a semantic priming paradigm exploring automatic access to the semantic system. Although AD patients showed a semantic deficit on the intentional semantic battery, they demonstrated normal semantic facilitation on the priming task. In the $\mathrm{AD}$ group as a whole, the semantic impairment did not preferentially affect the living category either in the intentional or automatic condition. Instead, a prevalent deficit for the living category was found in three AD patients ( $14 \%$ of the group) on the intentional semantic tasks, but not on the automatic one. These findings support the view that the category effect may not be a generalised phenomenon in AD but may be restricted to a limited number of patients. The intentional/automatic dissociation of the semantic breakdown demonstrated by AD patients is discussed in relation to different theories regarding the organisation of semantic memory.
\end{abstract}

(C) 2003 Elsevier Science Ltd. All rights reserved.

Keywords: Semantic system; Semantic priming; Living/non-living; Category effect

\section{Introduction}

The semantic memory deficit is an important component of cognitive decline in patients with Alzheimer's disease (AD) (Bayles \& Tomoeda, 1983; Martin \& Fedio, 1983). The breakdown of semantic memory is considered the main cause of their poor performance on a variety of tasks such as Naming, Verbal Fluency and Object Recognition tasks or tests probing knowledge of concept meaning (see Salmon, Butters, \& Chan, 1999 for a review). Although the semantic memory impairment is well documented even in the early stages of the disease, its exact characteristics are still a matter of debate. One of the most controversial issues is whether the semantic damage in AD preferentially affects a specific-category of knowledge. Contrasting data have been reported in this regard. In fact, some findings actually support a selective semantic impairment for

\footnotetext{
* Corresponding author. Tel.: +39-06-51501574; fax: +39-06-51501584.

E-mail address: r.perri@hsantalucia.it (R. Perri).
}

the categories of living beings (Daum, Riesch, Sartori, \& Birbaumer, 1996; Mazzoni, Moretti, Lucchini, Vista, \& Muratorio, 1991; Montanes, Goldblum, \& Boller, 1995; Silveri, Daniele, Giustolisi, \& Gainotti, 1991), while other studies report a homogeneous impairment of these two categories of knowledge (Gainotti, Di Betta, \& Silveri, 1996; Hodges, Salmon, \& Butters, 1992; Montanes, Goldblum, \& Boller, 1996; Tippett, Grossman, \& Farah, 1996) in AD patients.

Several different hypotheses have been advanced to account for the inconsistency of reported findings regarding the category effect in the semantic impairment of AD patients. Tippett et al. (1996) sustained that AD does not generally cause a selective category-specific deficit and that when such a deficit occurs it is likely an artefact due to imperfectly matched living/non-living stimuli sets. This claim is based on the finding that intrinsic properties of words such as familiarity, prototypicality and frequency of occurrence are generally lower for living than for non-living things (Funnell \& Sheridan, 1992; Hodges et al., 1992) and, in contrast, visual complexity is often greater for living than for 
non-living items (Gaffan \& Heywood, 1993). Accordingly, performance on living things is intrinsically "more difficult" than on non-living things and, therefore, is more susceptible to errors following brain damage. As a matter of fact, Tippett et al. (1996) demonstrated that if confounding factors inherent in tested items are equated, then differences in the performance of $\mathrm{AD}$ patients on living and non-living categories are no longer apparent.

Other authors have advanced different hypotheses to explain the inconstant presence of category effects in $\mathrm{AD}$ patients' semantic deficit. Garrard, Patterson, Watson, and Hodges (1998) and Gonnerman, Andersen, Devlin, Kempler, and Seidenberg (1997) posited that the living/non-living dissociation in the semantic memory impairment of AD patients is genuine but may affect individual patients in opposite ways. Thus, some patients may suffer from a prevalent difficulty with living beings and others may show the reversed pattern, that is, more severe impairment for non-living things. Therefore, in an AD sample when the number of cases with a prevalent impairment for living things prevails over cases with the opposite dissociation, then a significant effect with better average performance on non-living things is manifested. In contrast, when the proportion of cases showing the two opposite patterns of category dissociation is roughly comparable in an $\mathrm{AD}$ sample, then the group analysis fails to demonstrate any category effect.

Although Garrard et al. (1998) and Gonnerman et al. (1997) share the assumption that the semantic impairment in $\mathrm{AD}$ patients may result in a category dissociation that alternatively penalises living or non-living items, their interpretation of this phenomenon is different. According to Garrard et al. (1998), it is an expression of the variability of the AD pathology that most frequently prevails in the temporo-limbic regions, thus resulting in a prevalent impairment for the living category. However, occasionally it predominantly involves the fronto-parietal regions, thus accounting for the prevalent non-living deficit in a minority of AD patients. This claim is based on the hypothesis advanced by some authors (Gainotti, 1990; Silveri et al., 1991; Warrington \& Shallice, 1984) that the temporo-limbic structures are critically involved in the processing of perceptual information, supposed to be particularly important for the identification of living things, and, conversely, that the fronto-parietal regions are specialised for the representation of functional knowledge, assumed to be crucial for the identification of non-living things.

Instead, Gonnerman et al. (1997) rejected the explanations of category selective deficits in AD patients based on the role of different specialised anatomical regions and sustained that such category effects occur because of the intrinsic differences in the conceptual representation of living and non-living things within a connectionist model of semantic memory. Such models view semantic knowledge as a distributed and interconnected network of units representing different features of individual concepts. According to Devlin, Gonnerman, Andersen, and Seidenberg's (1998) and Gonnerman et al.'s (1997) models, living things are represented by many shared and inter-correlated semantic features (that is, features shared by several category members and that regularly co-occur in different concepts), while non-living things are represented by a greater proportion of distinctive features that are less correlated. In this kind of model, shared features are thought to be more resistant to degenerative damage than distinctive ones, because they are sustained by strong collateral support within a set of more inter-correlated features. Based on the assumption that category effects arise from damage to a unitary semantic system in which concepts in different categories are structured in different ways, the existence of selective deficits either for non-living or living things in AD patients is explained as a function of different stages of pathology. In the early stages of a widespread pathology such as $\mathrm{AD}$, the more vulnerable distinctive features are predominantly affected, thus resulting in a selective semantic deficit for non-living things. However, as the disease progresses, the degradation of entire sets of shared and inter-correlated features implies a catastrophic loss of knowledge of living things, thus producing a prevalent deficit for living items.

A final hypothesis regarding the inconsistent emergence of a category effect in the performance of $\mathrm{AD}$ patients on semantic memory tasks was proposed by Daum et al. (1996). These authors showed that discrepancies in experimental findings regarding AD patients' specific-category deficits could depend on the nature and, more specifically, on the degree of effortful processing required by the task. In their study, a group of $\mathrm{AD}$ patients performed worse on living than non-living items only on those tasks requiring an effortful access to semantic concepts, such as confrontation naming with and without cues, semantic knowledge probes or definitions of concepts. In other less demanding tasks, such as an object decision task (in which subjects were asked to decide whether a picture represented a real or an unreal object) or a preference judgement task (in which subjects were asked to express a preference between one real and one unreal item), AD patients not only performed better overall than on the previous tasks, but they performed at the same level on living and non-living items. Daum et al. (1996) interpreted these results in light of Nebes et al. proposal (Nebes, Brady, \& Huff, 1989; Nebes, Martin, \& Horn, 1984) that the semantic impairment in $\mathrm{AD}$ patients is due to the inability to consciously access or retrieve otherwise spared semantic information which, instead, can be available when implicitly assessed. Consequently, such a deficit, most probably related to generalised attention and executive deficits, may not be evident in low task demand conditions, in which semantic information can be accessed or retrieved automatically, but it becomes evident when the experimental paradigm calls for a more intentional (i.e. effortful) retrieval strategy.

A similar explanation can be proposed for the significant category effect found in the performance of AD patients in effortful but not in low demand semantic tasks. Indeed, since items belonging to living categories are intrinsically more 
difficult than non-living ones (Funnell \& Sheridan, 1992; Gainotti et al., 1996; Tippett et al., 1996), then intentionally accessing them may be particularly difficult for $\mathrm{AD}$ patients, thus giving rise to a specific-category effect. Instead, automatic access to semantic information, typical of low demand tasks, may overcome the AD patients' processing difficulties, thus obscuring any semantic category dissociation.

To summarise, this brief review of the literature highlights the different factors that may account for the inconsistent emergence of category-specific effects in the semantic impairment of $\mathrm{AD}$ patients. These include the following: (a) the different intrinsic characteristics of the living and non-living items utilised in the semantic tasks (Tippett et al., 1996); (b) the simultaneous presence of individuals dissociating in opposite directions in the selected AD patients' sample (Garrard et al., 1998; Gonnerman et al., 1997); (c) the different kinds of access to semantic knowledge, either automatic or intentional, required by the experimental task (Daum et al., 1996).

The aim of this study was to verify the influence such different factors may have on the emergence of category deficits in a sample of AD patients. In particular, intentional access to semantic knowledge was evaluated by means of a battery of tests that included Naming and Comprehension tasks and a Questionnaire exploring different levels of semantic knowledge. Data regarding frequency, prototypicality, visual complexity, name and image agreement and other potentially confounding factors were available for both the living and non-living items of the battery. This made it possible to analyse the performances of AD patients and normal subjects after partialling out the possible influence of these factors on performance accuracy. In order to investigate the presence of patients dissociating in opposite directions in the AD sample, a performance analysis of the individual cases as well as of the overall group was carried out. Finally, to evaluate automatic access to semantic knowledge, we used a lexical decision semantic priming task. Semantic priming is an experimental paradigm classically used to investigate the semantic store with a minimal demand for attentional resources. It consists of subjects' response times (for example, on Naming or Lexical Decision Tasks), which are faster to target words following a semantically related prime word than to target words following a non-related prime or a neutral prime (i.e. a string of XXX (Neely, 1977), or the word BLANK (Chenery, Ingram, \& Murdoch, 1994; Chertkow et al., 1994; Hartman, 1991)). Faster response time to related than to unrelated prime-target pairs is generally believed to reflect the spreading activation from the prime to the concepts that share a semantic relationship with it in the semantic store (Collins \& Loftus, 1975). The priming effect is automatic, that is it occurs rapidly and is not affected by preparatory strategies or expectations, when the interval between the presentation of the prime and the target, known as stimulus onset asynchrony (SOA), is brief $(<400 \mathrm{~ms})$, and the percentage of related prime-target pairs is low (usually around $20 \%$ of trials). Instead, intentional (i.e. strategy-based) attentional processes may contribute to semantic priming when longer SOAs or a higher proportion of related pairs are used (Neely, 1977, 1991). Besides facilitating the related condition compared to the unrelated and neutral ones, intentional priming paradigms also determine slower reaction times (RTs) for the unrelated than the neutral condition. This is because strategies based on the expectation of a related target inhibit response times to an unrelated target. Automatic priming paradigms, instead, produce only facilitation for the related condition with respect to the unrelated one, while response times to unrelated and neutral conditions do not differ.

In the present study, the primes of the associated pairs, alternatively belonging to living or non-living categories, were derived from the semantic battery; thus, intentional and automatic access to semantic knowledge was evaluated relative to the same items. Moreover, since we were interested in investigating non-intentional access to semantic knowledge, experimental parameters were set out to obtain an automatic form of priming (i.e. short SOA and low proportion of related prime-target pairs).

\section{Methods}

\subsection{Subjects}

Twenty-one subjects suffering from AD (15 females and 6 males; mean age $=70.7$ years, S.D. $=5.8$; mean years of education $=9.2$; S.D. $=4.7$ ) participated in this study. All subjects met the clinical criteria established by the National Institute of Neurological and Communicative Disorders and Stroke-Alzheimer's Disease and Related Disorders Association (McKhann et al., 1984) for probable Alzheimer's disease. Their medical history, neurological examination, brain imaging and laboratory tests provided assurance that their dementia symptoms could be not attributed to an illness other than $\mathrm{AD}$. The $\mathrm{AD}$ group was constituted by subjects with mild to moderate levels of dementia (mean score on the Mini mental state examination $=21.1$; S.D. $=2.9$; range 16.5-26.9; mean score on clinical dementia rating scale $=$ 1.1 ; S.D. $=0.4$; range $0.5-2$ ).

A group of 23 age- and education-matched normal controls (NCs) ( 17 females and 6 males; mean age $=68.0$ years, S.D. $=10.0$; mean years of education $=10.3$; S.D. $=5.0$ ) performed the semantic priming task; 13 of these subjects were also submitted to the intentional semantic battery.

Informed written consent was obtained from all participants and patients' caregivers prior to the study.

\subsection{Tasks}

\subsubsection{Battery for the evaluation of category-specific semantic deficits}

This battery, devised by Laiacona, Barbarotto, Trivelli, and Capitani (1993), consists of a pictures Comprehension 
task, a picture Naming task and a multiple-choice format Questionnaire. Six semantic categories are investigated, three of living things (fruits, vegetables and animals) and three of non-living things (vehicles, furniture and tools). The 60 linear drawings (10 from each of six categories) used as targets for the Naming task and as targets and distractors for the Comprehension task were taken from Snodgrass and Vanderwart's series (Snodgrass \& Vanderwart, 1980).

In the Comprehension task, subjects are asked to indicate the picture that corresponds to the one named by the experimenter out of five vertically placed alternatives. Distractors are four pictures belonging to the same semantic category (each pictures appeared an equal number of times as distractor). Each correct response scores 1 point (0-60).

For the Naming task, subjects are requested to name all 60 pictures; each correct response scores 1 point (0-60).

For each item of the verbal Questionnaire (e.g. apple) subjects are requested to answer six questions in a forced-choice response format with three alternatives. The first two questions pertain to superordinate information (one general superordinate (e.g. Is it an animal, a plant or an object?) and one in which the choice is within the living or non-living domain (e.g. Is it a tree, a vegetable or a fruit?)), two concern subordinate perceptual attributes (e.g. Is it round, oblong or conical?) and the last two concern subordinate functional attributes including any non-perceptual feature (e.g. Do we eat it raw, dried or in both ways?). Because of the length of the task, we decided to utilise only the 30 items corresponding to those used in the semantic priming task (see below). Each correct response scores 1 point (0-180).

Since performance on the semantic battery can be influenced by a number of intrinsic properties of the specific items, Laiacona and co-workers (Barbarotto, Capitani, \& Laiacona, 1996; Capitani, Laiacona, \& Barbarotto, 1993; Laiacona, Barbarotto, Trivelli, \& Capitani, 1993; Laiacona et al., 1993) suggested analysing performances by means of regression analyses in which response accuracy for single items of the Comprehension, Naming or Questionnaire tasks is co-varied for a number of intrinsic properties of words or pictures.

The word properties taken into account are:

(a) Frequency of the stimulus words in the Italian lexicon (Bortolini, Tagliavini, \& Zampolli, 1971) after logarithmic transformation.

(b) Prototypicality of the stimulus word according to Battig and Montague (1969). In their study, 442 normal controls were given a limited amount of time to name as many items as possible belonging to a given category. The frequency of each item was recorded as a measure of its prototypicality.

(c) Familiarity of the stimulus according to Snodgrass and Vanderwart (1980). Subjects were asked to rate how usual or unusual the concept of the given item was in their realm of experience on a 5 -point scale $(1=$ very familiar).
The following intrinsic properties of the pictures are considered:

(a) Name agreement, i.e. the percentage of times each name was given by the Italian control sample (Laiacona et al., 1993).

(b) Image agreement according to Snodgrass and Vanderwart (1980). Subjects were asked to indicate how closely each picture corresponded to their own mental image of the item on a 5 -point scale $(1=$ low agreement).

(c) Visual complexity according to Snodgrass and Vanderwart (1980). The amount of detail or intricacy of lines in the picture was judged on a 5-point scale $(1=$ very simple $)$.

Finally, since the difficulty of single questions on the Questionnaire varies in an unpredictable way, thus constituting another variable potentially able to influence response accuracy on single items, another intrinsic property of the items on this task was a difficulty index. This was obtained by asking 10 young, well-educated subjects to rate the difficulty of each question on a 5 -point scale $(1=$ very difficult $)$ (Laiacona et al., 1993).

Regarding the Naming and Comprehension tasks, there is significantly lower familiarity for living than non-living things $(t=3.73, P<0.001)$; there is also a trend for livings things to have a lower occurrence frequency value $(t=$ $1.83 ; P=0.07)$ and a higher image agreement value $(t=$ 1.86; $P=0.07)$; the difference between items in the two semantic domains did not approach statistical significance for any of the other variables. Regarding the Questionnaire (for which the number of items was halved), there was a nearly significant lower level of difficulty $(t=1.91 ; P=$ $0.06)$ for items referring to the non-living category. The difference between the other variables in the two semantic domains did not approach significance.

\subsubsection{Semantic priming on lexical decision task}

Two semantic priming experiments were developed, one for the living and one for the non-living category.

2.2.2.1. Material. The 60 items of the previous semantic battery were utilised to create the semantically related word/word pairs. For this purpose, we submitted the entire list of 60 words constituting each item of the semantic battery to 60 university students. The lists, printed in columns on three sheets of paper, corresponded to three different random orders of the words. For each word, the subjects were requested to write down the first associate that came to mind.

Thirty related prime-target pairs were created by associating a word (target) chosen from the three most frequently produced by the 60 university students for that specific item with each word on the list (prime). Fifteen primes belonged to the living category and 15 to the non-living one. The 30 associated targets concerned both category co-ordinates (e.g. onion-garlic) and perceptual or functional properties 
(e.g. pepper-red or train-trip) of the primes in comparable numbers for each category. The equal presence in the two categories of prime-target pairs linked by these two levels of semantic knowledge (co-ordinate and attribute) made it possible to exclude that the differences in the priming effects between the two domains were due to the different number of properties shared by the prime and the target in the two living and non-living sets of items (Giffard et al., 2002). As previously noted, prime words of the semantic priming task were also used as stimulus items for the Questionnaire task. Accordingly, prime words for the living and non-living pairs did not differ for frequency of occurrence, familiarity and prototypicality. Living and non-living pairs were also chosen so that they were comparable for frequency of association (mean number of subjects who produced that association for living pairs $=22.4$, S.D. $=7.7$; for non-living pairs $=20.9$, S.D. $=11.2$ ). Finally, target words were comparable for frequency of occurrence in the Italian lexicon (living: mean 34.6 per million, S.D. 45.5; non-living: mean 46.9 per million, S.D. 48.7) and length (all words ranged from 3 to 10 letter with a mean of 5.33 letters for the living condition (S.D. 1.23) and 5.6 letters (S.D. 1.35) for the non-living condition).

For the experiment with living things, 15 unrelated word/word pairs were created using the target words of the associated pairs and replacing the prime with a different unrelated word. The latter also belonged to a living category, matched for frequency and length with the prime word. Fifteen neutral word/word pairs were constituted by the word NULLA (BLANK in Italian) as prime and the same target as the associated pairs. We utilised the word NULLA in the prime position instead of a string of XXX to avoid attentional problems in the shift from linguistic to non-linguistic processing during the task (see Silveri, Monteleone, Burani, $\&$ Tabossi, 1996 for a discussion of this issue).

Thirty word/legal-pseudoword pairs were created using the prime words of the previous related and unrelated stimuli in the prime position, and 15 word/legal-pseudoword pairs were created using the word NULLA in prime position. Legal-pseudowords were created by replacing one or two letters of real words.

Finally, to increase the total number of stimuli, 30 filler pairs, comprised of 15 unrelated word/word pairs and 15 word/legal-pseudoword pairs, were created. The related pairs represented $12.5 \%$ of the total number of word/word pairs. The probability of encountering a word versus a pseudoword in the target position was $50 \%$.

With the same criteria described for the experiment with living things, 15 unrelated word/word pairs, 15 NULLA/ word pairs, 30 word/pseudoword pairs, 15 NULLA/ pseudowords pairs, 15 word/word filler pairs and 15 word/ pseudoword filler pairs were created for the non-living experiment. Also in this case, the related pairs represented $12.5 \%$ of the total number of word-word pairs and the probability of encountering a word rather than a pseudoword in the target position was $50 \%$.
For both the living and non-living experiments, stimuli were divided into 3 blocks of 40 pairs per block containing an equal number of Related, Unrelated and Neutral pairs, an equal number of word/pseudoword pairs and filler pairs. A particular target word appeared three times in the Related, Unrelated and Neutral condition, always in a different block. The computer program randomised the order of presentation of the stimulus pairs inside a particular block. The order of presentation of each block varied in a pseudorandom fashion from subject to subject.

2.2.2.2. Procedure. The experiments were run individually and controlled by a PC 386 . The stimuli were presented in white capital letters in the centre of a black computer screen and occupied about 5.7 degrees of visual angle. Each trial started with the presentation of a small central cross for $2000 \mathrm{~ms}$, indicating the fixation point. The cross was then followed by the prime word, which remained for $300 \mathrm{~ms}$ and was immediately replaced by the target, which remained until the subject responded or, in the case of lack of response, for $5000 \mathrm{~ms}$ and was then replaced by the empty black computer screen. The experimenter controlled the beginning of each trial by pressing a key on the computer keyboard. Subjects were placed approximately $50 \mathrm{~cm}$ from the computer screen and were instructed to silently read the prime and to perform a lexical decision task on the target. They had to press a red button for words and a green button for pseudowords with their dominant hand (the right hand for all patients and control subjects who participated in this study). The red button was always placed to the right of the subject and the green button to the left. During the experiment, subjects had to keep their dominant hand between the two buttons and move it towards one or the other at the moment of responding. If the subjects pressed the wrong button, the mistake was indicated by an auditory signal. The PC recorded response reaction times and accuracy. Performing the entire semantic priming task required about $30 \mathrm{~min}$. Half of the subjects performed the experiment with living things first and then with non-living things; the other half performed the experiments in the reverse order. There was an interval of $10 \mathrm{~min}$ between the two semantic priming experiments. Instead, there were no intervals between blocks within each experiment.

To ensure that each patient was able to perform the semantic priming task, the experimental session was preceded by a reading and a pre-test priming task. The reading task consisted of 20 words, matched for frequency and length to those of the experimental stimuli. Each word was presented in white capital letters on the centre of the black computer screen for $300 \mathrm{~ms}$, preceded by a small central cross for $2000 \mathrm{~ms}$. The pre-test priming task consisted of 10 word/word pairs and 10 word/legal-pseudoword pairs, with the same characteristics of length and frequency as the experimental pairs; they were presented with the same procedure as the experimental task. This task was preceded by some practice trials. Patients who were not able to read at least 
18 words in the reading task and to make at least 18 correct lexical decisions in the pre-test priming task were excluded from the study. Of the 43 patients initially recruited for the study, only the 21 from the experimental group reached the cut-off score on both pre-experimental tasks and were included in the study.

The recruitment requirement of a high performance level on the lexical decision task was to ensure that no deficits in the priming effect could be attributed to the AD patients' inability to access the semantic store through a degraded input lexicon. Indeed, although lexical decision tasks can be performed only by relying on lexical (i.e. non-semantic) knowledge, the facilitation that primes produce on the lexical decision latency for related targets can be assumed to be semantically mediated. According to interactive spreading activation models of language (McClelland \& Rumelhart, 1981), which assume a continuous feedback between more central and peripheral components of language mechanisms, the prime-related target facilitation should be based on the partial activation of the target in the input lexicon due to the number of features that it shares with the prime at the semantic level (Chertkow \& Bub, 1989).

2.2.2.3. Testing sessions. Each subject was submitted to two testing sessions: in the first session, the entire intentional semantic battery was administered (the order of administration of the Comprehension, Naming and Questionnaire tasks was counterbalanced between subjects); in the second one (the next day) the semantic priming experiments.

\section{Results}

\subsection{Battery to evaluate category-specific semantic deficits}

\subsubsection{Comparison of group performances}

Mean performance accuracy obtained by $\mathrm{AD}$ patients and NCs on the living and non-living items of the Naming, Comprehension and Questionnaire tasks are shown in Table 1 (one patient in the AD group did not fill in the Questionnaire, so the results for this task are averaged on 20 patients).

\subsubsection{Naming and Comprehension}

Performance scores for Naming and Comprehension tasks were submitted to a two-way MANOVA with Group (AD patients and NCs) as between factor and Category (living and non-living) as within factor. In each task, a significant Group effect emerged (Naming: $F=9.5, P<0.05$ with 1, 32 d.f.; Comprehension: $F=6.5, P<0.05$ with 1 , 32 d.f.). However, both the Category effect ( $F=2.1$ with 1,32 d.f. and $F=0.4$ with 1,32 d.f. for the Naming and Comprehension tasks, respectively) and the Group $x$ Category interaction $(F=0.3$ with 1,32 d.f. and $F=$ 0.8 with 1,32 d.f., respectively) were non-significant in the two tasks. This result shows that, although AD patients performed worse than NCs on Naming and Comprehension tasks, the two groups performed at the same level on both tasks on living and non-living items.

\subsubsection{Questionnaire}

Since some authors have suggested that the semantic degradation in $\mathrm{AD}$ patients may mostly affect the distinctive features of concepts, while information shared by several concepts is relatively spared (Gonnerman et al., 1997; Moss, Tyler, Durrant-Peatfield, \& Bunn, 1998), the analysis of the Questionnaire scores considered the mean performance accuracy obtained by $\mathrm{AD}$ patients and NCs separately on the superordinate and subordinate questions. To equate the number of responses obtained on the two types of questions, the subordinate ones were halved. A three-way MANOVA was performed with Group (AD patients and NCs) as between Factor and Category (living and non-living) and Type of question (superordinate and subordinate) as within factors. The Group effect approached significance $(F=3.9, P=0.057$, d.f. 1,31$)$; infact, $\mathrm{AD}$ patients (average accuracy: 27.39) performed substantially worse than NCs (average accuracy: 29.31). The Category ( $F=20.6, P<0.001$ with 1,31 d.f.) and the kind of question effects $(F=7.2, P<0.01$, d.f. 1,31$)$ were also significant, due to the better performance on non-living (mean 28.96) than on living items (mean 27.75) and on superordinate (mean 28.83) than on subordinate questions (mean 27.87) by the overall group of subjects. The lack of a significant Group $\times$ Category interaction $(F=20.6$ with 1,31 d.f.) indicated that performance on living items was equally more difficult for AD patients and NCs than

Table 1

Mean (and S.D.) scores obtained by AD patients and NCs on Naming, Comprehension and Questionnaire (superordinate and subordinate questions) for both living and non-living condition

\begin{tabular}{llllll}
\hline Group & Category & Naming $(\max 30)$ & Comprehension $(\max 30)$ & & Questionnaire \\
\cline { 3 - 6 } & & & & Superordinate $(\max 30)$ & Subordinate $(\max 30)^{\mathrm{a}}$ \\
\hline AD & Living & $21.9(6.9)$ & $28.2(2.7)$ & $27.5(2.9)$ & $25.6(4.8)$ \\
& Non-living & $23.3(6.9)$ & $28.8(1.7)$ & $28.8(2.5)$ & $27.5(4.5)$ \\
\multirow{2}{*}{ NCs } & Living & $27.9(1.8)$ & $30.0(0.0)$ & $29.0(1.5)$ & $28.8(1.6)$ \\
& Non-living & $28.5(1.1)$ & $29.9(0.2)$ & $29.9(0.6)$ & $29.5(0.7)$ \\
\hline
\end{tabular}

\footnotetext{
${ }^{a}$ The mean scores were halved in this condition in order to equate them to the superordinate ones.
} 
performance on non-living ones. Instead, a trend toward a significant interaction between Group $\times$ Type of question emerged ( $F=3.3, P=0.08$, d.f. 1,31$)$. Indeed, while AD patients performed at a level comparable to that of NCs on the superordinate questions (average accuracy $=28.2$ and 29.4 , respectively) $(F=2.7, P=$ n.s. with 1,31 d.f. $)$, they performed worse than NCs on the subordinate questions (average accuracy $=26.5$ and 29.1, respectively) $(F=4.2$, $P=0.05$, with 1,31 d.f.). The lack of any other significant interaction indicated that the relative sparing on the superordinate and the impairment on the subordinate questions in the $\mathrm{AD}$ group was present equally in the two semantic categories of knowledge.

\subsubsection{Multiple regression analyses of group performances}

As previously discussed, a number of intrinsic properties of words (i.e. Frequency, Familiarity and Prototypicality) and pictures (Name Agreement, Image Agreement and Visual Complexity) and also the difficulty rate of single questions on the Questionnaire can influence performance accuracy on the semantic battery tasks. Some of these variables assume substantially different average values in semantic battery items belonging to living or non-living categories. To assess the actual role played by the living/ non-living dichotomy in predicting performance accuracy on single items, and to rule out the possible confounding influence of intrinsic properties of pictures, words and questions, Capitani et al. (1993) suggested analysing performance on these tasks by means of regression analyses.

Therefore, separate stepwise multiple regression analyses were first performed for the AD and NC groups and for each of the semantic tasks. In each of these analyses, the average successful Naming, Comprehension or Question response to single items achieved by the whole group was the dependent variable and living or non-living category (coded as dummy variable (Kerlinger \& Pedhazur, 1973)), Frequency, Prototypicality and Familiarity of the individual items were the independent variables. Additional independent variables for the Naming and Comprehension tasks were Name Agreement, Image Agreement and Visual Complexity, and for the Questionnaire they were difficulty index of individual questions and type of question. Since the statistical units in each of these regression analyses were represented by the task items (60, 60 and 180 for Comprehension, Naming and Questionnaire, respectively), there was a ratio of 7.5 cases for each variable introduced for the regression analyses performed on Comprehension and Naming tasks, and a ratio of 30 cases for each variable studied in the Questionnaire analysis. These ratios were above the minimum ratio of at least five cases per variable usually requested in multiple regression analyses (Hair, Anderson, Tatham, \& Balch, 1998).

For both Naming and Comprehension tasks, the category of the individual items did not contribute to the prediction of response accuracy for either the $\mathrm{AD}$ or the $\mathrm{NC}$ group.
As for the performance of $\mathrm{AD}$ patients on the Questionnaire, the first independent variable to enter in the equation was question difficulty with a multiple $R$ of $0.50\left(R^{2}\right.$ change $=0.25 ; F=62.1 ; P<0.001)$. In the second step of the regression analysis, a significant contribution toward predicting the dependent variable was made by the living/non-living dichotomy with a multiple $R$ of $0.55\left(R^{2}\right.$ change $=0.05 ; F=13.1 ; P<0.001)$. When forced to enter the regression equation, none of the remaining independent variables contributed significantly toward predicting the dependent variable. Substantially similar results were obtained with NCs. Also in this case, the first independent variable to enter the equation was question difficulty with a multiple $R$ of $0.28\left(R^{2}\right.$ change $=0.08 ; F=15.6$; $P<0.001)$ followed by the living/non-living dichotomy in the second step $\left(R=0.32 ; R^{2}\right.$ change $=0.02 ; F=4.4$; $P=0.036)$. No other independent variables entered the equation.

\subsubsection{Logistic regression analyses of single subject's performance}

Gonnerman et al. (1997) and Garrard et al. (1998) proposed that a specific-category deficit might not be evident in the $\mathrm{AD}$ group because of a possible double dissociation with some patients showing the expected prevalent impairment for the living category and others the opposite dissociation penalising the non-living category. In order to highlight possible individual dissociations, we submitted individual performances of subjects in the AD and NC groups on the Naming, Comprehension and Questionnaire tasks to logistic regression analysis (Laiacona et al., 1993). In each of these analyses, the dependent variable was represented by the performance accuracy of the individual patient on the single items (coded as 0 or 1 ) while the same intrinsic properties of words, pictures and questions considered before in the multiple regression analyses were the co-variates. One $\mathrm{AD}$ patient (CE) showed a significant category effect on all three tasks with disproportionately worse performances on living items than on non-living ones; another patient (DE) performed significantly worse on living than on non-living items on Naming and on the Questionnaire; and four patients (CL, GR, MA and TR) showed a category effect with poorer performances on living than on non-living things only on the Questionnaire.

The logistic regression analyses applied to individual NCs' performances revealed that in no case did the living/non-living dichotomy significantly contribute to the prediction of Naming and Comprehension performances. Instead, the category effect was significant for predicting performance on the Questionnaire in three cases with performance on living items consistently worse than on non-living ones.

In order to verify whether the disadvantage for living items shown by the six AD patients was the same as that displayed by the NCs or whether it was disproportionately larger, we computed the $t$ scores for each subject relative 
to the living/non-living independent variable resulting from the logistic regression analyses made on the performance of each NC subject on the three tasks. The $t$ score can be considered a standardised index of each subject's trend to perform better in one category than the other because it takes into account the confounding effects exerted by intrinsic properties of words and pictures. The direction of the $t$ (positive or negative) is indicative of the category in which the subject performed better (living or non-living, respectively) and the magnitude of the $t$-value is indicative of the entity of this advantage ( $t=0$ corresponding to an identical performance in the two categories). We utilised the mean of the $t$ scores obtained by NCs +3 S.D. in the Naming and Questionnaire tasks as the normal cut-off of the advantage on non-living items with respect to living ones on these two tasks (NCs' Naming mean $t$ score $=0.43$, S.D. 0.53, cut-off 2.08; NCs' Questionnaire mean $t$ score $=0.43$, S.D. 0.77, cut-off 2.74) (the Comprehension task was not considered in this case because all NCs performed at or close to the ceiling). Such a high cut-off of normality was adopted to ensure that the AD patients' category asymmetries above the normal cut-off were not simply due to chance as a consequence of the high number of logistic regressions performed on the individual data. On the Naming task, the two AD patients with a significant disadvantage for living things in the regression analysis both had a $t$ score above the normal cut-off
(CE: $t=2.36$ and DE: $t=2.36$ ); moreover, two of the six patients with a significant disadvantage for living things in the regression analysis of the Questionnaire data also had a $t$ score above the normal cut-off (CE: $t=3.04$ and GR: $t=3.02)$. To conclude, in three AD patients of our sample $(14 \%)$ the disadvantage for living things exceeded what we expected simply based on the normal subjects' performance.

\subsection{Relationship between category dissociation and severity of cognitive and lexical semantic impairment}

Since some authors have suggested that the severity of the AD pathology may be predictive of the presence of category dissociation (Gonnerman et al., 1997), we wanted to verify the role of the overall level of mental deterioration or lexical-semantic impairment in the emergence of the category effect in our sample of AD patients.

Fig. 1 shows the distribution of individual AD patient's $t$-values for the category effect on the Naming and Questionnaire tasks (as resulting from the logistic regression analyses), as a function of their MMSE score or overall accuracy scores on those tasks. In each case, the three AD patients who showed a specific disadvantage for the living category fell on the side of the median corresponding to more severe impairment as measured by the distribution of MMSE or overall Naming and Questionnaire accuracy scores.

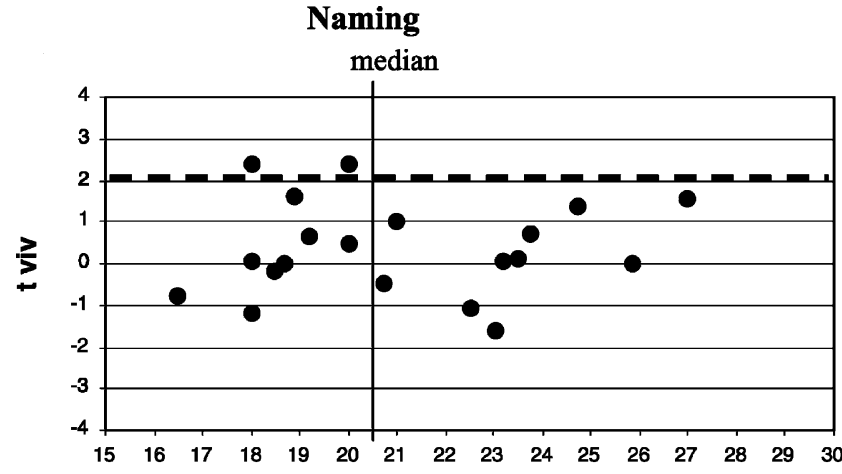

(a)

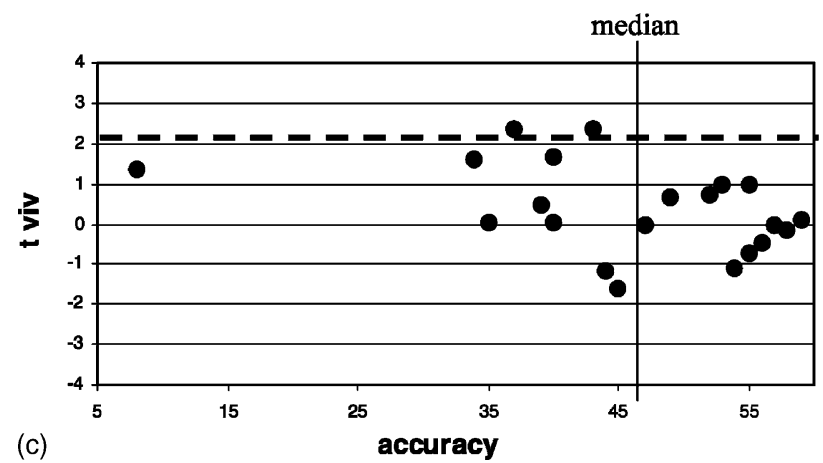

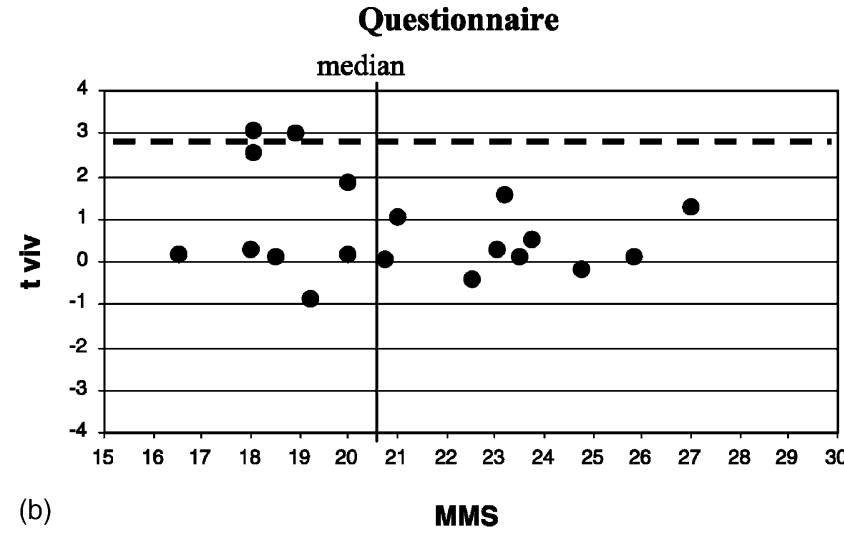

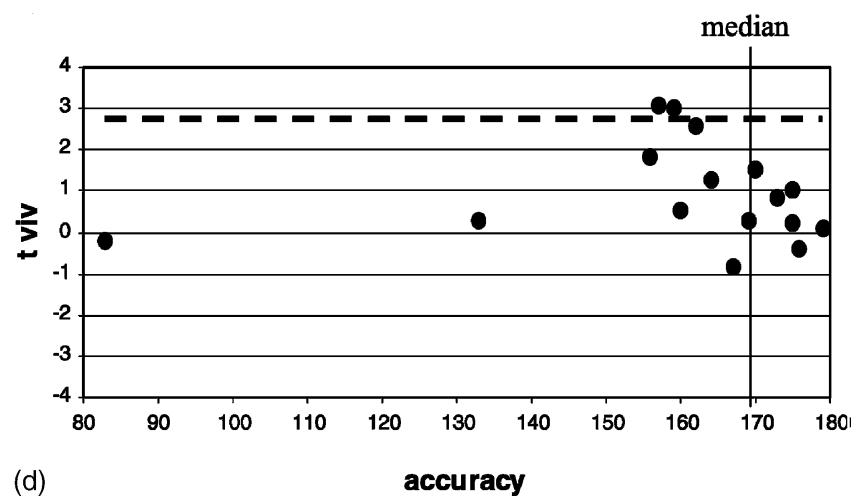

Fig. 1. Distribution of individual AD patients' $t$-values for the category effect on the Naming (panels a and b) and Questionnaire (panels $c$ and d) as a function of their MMSE score or overall accuracy scores on those tasks. Dot lines show the cut-off scores considered as normal for the disadvantage on living items. 
Table 2

Mean (and S.D.) of RTs and accuracy scores obtained by AD patients and NCs on Related, Unrelated and Neutral pairs in the semantic priming task both for living and non-living condition

\begin{tabular}{|c|c|c|c|c|c|c|c|}
\hline \multirow[t]{2}{*}{ Group } & \multirow[t]{2}{*}{ Category } & \multicolumn{3}{|c|}{ Accuracy $(\max =15)$} & \multicolumn{3}{|l|}{ RTs (ms) } \\
\hline & & Related pairs & Unrelated pairs & Neutral pairs & Related pairs & Unrelated pairs & Neutral pairs \\
\hline \multirow[t]{2}{*}{$\mathrm{AD}$} & Living & $13.7(1.2)$ & $13.0(1.5)$ & $13.6(1.3)$ & $1328.3(314.6)$ & 1347.5 (281.1) & $1300.7(268.8)$ \\
\hline & Non-living & $12.9(1.9)$ & $13.0(1.9)$ & $13.0(1.8)$ & $1315.6(312.4)$ & $1390.4(387.9)$ & $1356.1(332.6)$ \\
\hline \multirow[t]{2}{*}{$\mathrm{NCs}$} & Living & $13.6(1.3)$ & $13.7(1.3)$ & $14.1(1.3)$ & $927.3(323.1)$ & $957.2(388.2)$ & $953.2(353.0)$ \\
\hline & Non-living & $13.9(1.1)$ & $13.8(1.2)$ & $13.6(1.1)$ & $941.3(375.2)$ & $1000.0(450.4)$ & $988.4(470.7)$ \\
\hline
\end{tabular}

\subsection{Semantic priming on lexical decision}

\subsubsection{Accuracy}

The average number of correct lexical decisions made by $\mathrm{AD}$ patients and NCs on the target words of the associated pairs in both the living and non-living semantic priming experiments and as a function of being preceded by a Related, Unrelated or Neutral prime are reported in Table 2. These data were submitted to a three-way MANOVA with Group (AD patients and NCs) as between factor, and Priming Condition (Related, Unrelated and Neutral) and Category (living and non-living) as within factors. The difference in accuracy between groups approached significance $(F=2.9$, $P=0.09$ with 1,41 d.f.). However, the Priming Condition, Category effects and interactions were not significant, probably due to a ceiling effect of performance.

\subsection{Response reaction times}

Only reaction times on correct lexical decisions were considered for statistical analysis. In addition, trials with RTs longer than 2 S.D. from each individual's mean RT for that condition were excluded from all the statistics. The average RTs obtained by AD patients and NCs as a function of Priming Condition and Category are reported in Table 2. These data were submitted to a three-way MANOVA with Group (AD patients and NCs) as between factor and Priming Condition (Related, Unrelated and Neutral) and Category (living and non-living) as within factors. The results showed significant effects of Group $(F=13.4, P<0.001$ with 1,42 d.f.) and Priming Condition $(F=4.8, P<0.01$ with 1,42 d.f.), but not of Category ( $F=1.1$, with 1,42 d.f.). AD patients showed slower RTs (mean $1339 \mathrm{~ms}$ ) than NCs (mean $961 \mathrm{~ms})(F=13.4, P<0.001$ with 1,42 d.f. $)$ and, in the overall group, lexical decision in the Related condition was significantly faster (mean $1128 \mathrm{~ms}$ ) than in the Unrelated one (mean $1173 \mathrm{~ms})(F=8.1, P=0.006$ with 1,42 d.f.). The Neutral condition (mean $1149 \mathrm{~ms}$ ) did not differ from either the Related or Unrelated ones $(F=2.2$ with 1,42 d.f. and $F=3.1$ with 1,42 d.f., respectively). The lack of any inhibitory effect in the Unrelated condition with respect to the Neutral one meets the generally accepted operational criterion to consider the lexical decision facilitation exerted by a semantic priming paradigm as automatically generated
(Neely, 1977). The non-significant Group $\times$ Priming Condition interaction ( $F=0.7$ with 1,42 d.f.) documented a similar magnitude of the priming effect in the AD (46 ms) and NC (44 ms) groups. The Priming Condition $\times$ Category interaction was not significant ( $F=2.1$ with 1,42 d.f.). However, the priming effect for the living category $(25 \mathrm{~ms})$ was substantially smaller than that for the non-living items (67 ms) $(F=3.1, P=0.08$ with 1,42 d.f. $)$. Finally, the three fold Group $\times$ Priming Condition $\times$ Category interaction fell short of significance ( $F=0.3$, with 1,42 d.f.), thus documenting that such a discrepancy of priming as a function of semantic category was of the same magnitude in both $\mathrm{AD}$ and $\mathrm{NC}$ subjects.

To verify whether the three AD patients who showed a specific disadvantage for the living category on the tasks of the intentional semantic battery also displayed reduced priming for living items, a measure of inter-category discrepancy was computed for each subject. The magnitude of priming in the pairs of the non-living category was subtracted from that of the living pairs. Analogously to what we previously made for the tasks of the semantic battery, a normality cut-off for this inter-category discrepancy was calculated by adding to the mean difference in priming obtained by NCs $(-28.78$ ms) 3 S.D.s $(92.68$; cut-off -306.8$)$. All three patients showed a discrepancy between priming for living and non-living pairs $(33,142.3$ and $-105 \mathrm{~ms}$, respectively) that was largely within the normal range.

\section{Discussion}

Aim of this study was to verify the possible influence of the following factors on the emergence of category effects in AD patients: (a) the different level of difficulty of the living and non-living items utilised in the semantic tasks (Tippett et al., 1996), (b) the simultaneous presence of opposite category-specific semantic deficits in the AD patients of the samples studied (Garrard et al., 1998; Gonnerman et al., 1997) and (c) the different modality of access (automatic or intentional) to semantic knowledge (Daum et al., 1996).

To achieve this, the performance of AD patients and NCs was analysed on a test battery investigating intentional access to semantic knowledge by controlling for the influence of several confounding factors inherent in the intrinsic 
properties of the items utilised. Three main results emerged from this analysis: (1) the finding of a "physiologic" category asymmetry (i.e. poorer performance on living than on non-living items in healthy subjects) (2) the lack of any category effect exceeding the asymmetry found in NCs in the performances of the AD group as a whole and (3) the presence of such a specific-category impairment in $14 \%$ of the individual patients of the overall $\mathrm{AD}$ sample. $\mathrm{AD}$ patients and NCs were also submitted to an automatic semantic priming experiment investigating automatic access to the same items as those of the intentional battery. The normal priming displayed by the $\mathrm{AD}$ group suggested a dissociation between impaired intentional access and normal automatic processing of semantic knowledge. Such a dissociation was evident in the AD group as a whole and in the AD patients who showed a significant category effect.

\section{1. "Physiologic" category asymmetry}

Although a disadvantage for the living category was only nominal in the Naming task and absent in the Comprehension task (in which NCs generally approached the maximum score), a category-based asymmetry penalising the items of the living category was revealed by the performances of NCs on the Questionnaire. Moreover, a trend toward a "physiologic" disadvantage for the living category was also found when automatic processing was requested in the semantic priming task.

The finding of a poorer performance on living than on non-living items in healthy subjects was not unexpected. It was reported by Capitani, Laiacona, Barbarotto \& Trivelli (1994) on the same Naming and Questionnaire tasks used in this study. A first explanation of this phenomenon involves the generally held assumption that items of the living categories are more difficult than those of the non-living ones (Funnell \& Sheridan, 1992). Indeed, in our corpus of words living items were less familiar and less frequent than non-living ones. Moreover, on the Questionnaire, queries about living items were on average more difficult than questions about non-living things. However, the category effect survived also when we analysed the NCs' performances by means of regression analyses to partial out these confounding factors. Obviously, the possibility remains that other factors might have made items belonging to the living category more difficult to process. For example, we did not consider Age of Acquisition or Imageability of the items used in the semantic battery (even though previous studies have documented that these factors favour living things (Lambon Ralph, Howard, Nightingale, \& Ellis, 1998; Morrison, Chappell, \& Ellis, 1997)).

A different explanation of the "physiologic" category asymmetry derives from recent claims of discrepancies in the way semantic representations regarding living and non-living concepts are stored in the human brain (Devlin et al., 1998; Gonnerman et al., 1997). Recently, Garrard, Lambon-Ralph, Hodges, \& Patterson (2001) reported a different distribution of the feature correlations across categories. Since living things have many more correlated features than non-living things, this fact could have consequences on the semantic system in both normal and impaired circumstances (see Garrard et al. (2001) for a detailed discussion of this issue). Furthermore, McRae, De Sa, \& Seidenberg (1997) demonstrated that in a semantic priming task, the magnitude of item by item facilitation was predicted by similarity between concepts expressed in terms of individual features for non-living things and, conversely, by similarity expressed in terms of correlated feature pairs for living beings. To summarise, the presence of a qualitative difference in the semantic representation of living and non-living concepts could be another explanation (not necessarily alternative to the discrepant difficulty argument) of the living disadvantage exhibited by NCs when they access their semantic knowledge either intentionally or automatically.

\subsection{Category effect}

Although the AD patients examined in this study were impaired on all tasks of the semantic battery, the group of patients as a whole failed to show a category asymmetry beyond the one exhibited by NCs.

The lack of a category effect in our AD sample can not be explained by the hypothesis that in an unselected group of AD patients an overall category effect is obscured by the simultaneous presence of individual cases with opposite patterns of dissociation (Garrard et al., 1998; Gonnerman et al., 1997). Indeed, while we found some individual cases showing a specific disadvantage for living things, in no case was the opposite dissociation observed. This is in striking contrast with Garrard et al.'s, 1998 study, which reports a significant disadvantage for non-living things in about $10 \%$ of the AD sample. One reason for this discrepancy may be the different degree of cognitive deterioration affecting the two AD groups. In Garrard et al.'s study (Garrard et al., 1998), all patients who showed a specific-category deficit had an MMSE score of 16 or less. In the present study, the selected AD population had a mean MMSE score of 21.1, with a range of 16.5-26.9. This sampling bias may have prevented the emergence of more cases with a disadvantage for living things and, perhaps, even of cases with a specific disadvantage for artefacts (indeed the three AD patients with a specific living impairment were among the most deteriorated in our sample). On the other side, the mild degree of dementia in our AD sample may also have been responsible for the lack of a specific deficit for the living category in the overall group. Such considerations are largely supported by the results of a recent study by our group (Zannino, Perri, Carlesimo, \& Caltagirone, 2002).

\subsection{Intentional versus automatic access}

Results indicate that our group of AD patients had intact semantic priming. Since the same stimuli were used in both 
the semantic priming task and the intentional semantic battery, the dissociation in $\mathrm{AD}$ patients between normal priming and impaired performance on all tasks of the semantic battery might not be an artefact due to imperfectly matched stimuli sets. Regarding category effects, the primary focus of the present study, we found that the AD patients did not show any specific disadvantage for the living category in the semantic priming task. This result was expected because of the absence of a specific-category effect also in the intentional semantic battery in the overall group of AD patients. However, the three patients who showed a significant disadvantage for the living category on the intentional semantic battery did not show a greater discrepancy between priming on living and non-living pairs than the NCs.

Several previous studies have reported intact automatic priming in AD patients (Hodges et al., 1992; Nebes et al., 1984; Ober, Shenaut, Jagust, \& Syillman, 1991). Different hypotheses have been advanced to explain this finding. On the assumption that information that does not exist cannot be primed (Shallice, 1988), the presence of preserved semantic priming in $\mathrm{AD}$ patients has been considered indicative of spared semantic knowledge by some authors (Hodges et al., 1992; Nebes et al., 1984; Ober et al., 1991). Nebes et al. (1984) suggested that although semantic knowledge is preserved in AD, it is not readily accessible or usable if the cognitive processes involved in a task make heavy demands on attentional capacity (i.e. if the task requires intentional retrieval), while, if the task calls primarily for automatic processing, AD patients perform fairly normally. Accordingly, the dissociation in our study between normal priming and poor performance on the semantic battery, could be interpreted as reflecting the discrepancy between normal automatic and deficient deliberate access to semantic information.

On the basis of Daum et al.'s findings (Daum et al., 1996), we hypothesised that an access semantic deficit might also be responsible for the emergence of specific-category effects in the $\mathrm{AD}$ group on intentional but not on automatic semantic tasks. We reasoned that in tasks relying on intentional access to semantic information, the decreased attentional capacities of AD patients would mostly affect the more difficult items (that is, the living ones, as suggested by data in the literature (Funnell \& Sheridan, 1992; Gainotti et al., 1996; Tippett et al., 1996) and confirmed in this study). However, when the task provided automatic access to semantic knowledge, not only were the AD patients' overall performances normal, but also the discrepancy in performance level between categories was no longer detectable. The fact that the three AD patients in our sample who showed a significant disadvantage for living items on the tasks of the intentional semantic battery had an inter-category priming discrepancy falling in the range of the NCs, is substantially consistent with this hypothesis.

The claim that a normal priming is indicative of substantially spared semantic knowledge is not, however, universally accepted. In some authors' opinion (Rapp \&
Caramazza, 1993), the same performance pattern, namely, an impairment on traditional semantic memory tasks but a normal semantic priming effect, can also be expected if a partial degradation of the semantic representation is assumed. In connectionist models of semantic memory, retrieval of a known concept implies activation of a specific pattern of processing units across the network that represents various attributes of that concept (Farah \& McClelland, 1991; Rumelhart \& McClelland, 1986). On the assumption that semantically related concepts have similar patterns of activation, these models make it possible to conceptualise the facilitation effect that occurs in semantic priming tasks as resulting from the activation of overlapping patterns of units (Masson, 1995; Parasuraman \& Martin, 1994). In this context, some authors (Rapp \& Caramazza, 1993) hypothesise that damage to stored information in the semantic network may involve elevation of unit activation thresholds. In this case, if the presentation of a single stimulus (as in the naming tasks) is not able to drive the corresponding units above the threshold activation levels, the presentation of a prime, sharing several units with the stimulus target, may increase the activation level of the damaged units to above-threshold levels and, therefore, may produce a facilitation effect. In this view, it is possible that the normal priming effect observed in AD patients is due to partially damaged, but not completely lost, semantic information. This supports the view (as in the intentional versus automatic access hypothesis) that the dissociation between normal and impaired semantic performance in AD patients depends on different kind of tasks adopted to assess their semantic memory.

Recent theories postulating that the degenerative process in $\mathrm{AD}$ pathology does not affect all semantic representation of a given concept to the same extent provide an alternative interpretation for our data (Devlin et al., 1998; Gonnerman et al., 1997; Moss et al., 1998). Based on these theories, the dissociation in $\mathrm{AD}$ patients' semantic performance may be an expression of the particular portion of the semantic representation tapped by the particular task. In the context of connectionist distributed models of semantic memory, it has been argued that in a widespread pathology such as AD, the unique or non-overlapping units of the semantic network are more precociously affected than the overlapping ones (Parasuraman \& Martin, 1994). According to the hypothesis of a priming effect based on the activation of overlapping features of two related concepts (Masson, 1995), normal facilitation can be interpreted as the expression of preserved functioning of the more resistant portion of the network in $\mathrm{AD}$ patients. On the other hand, since naming and word-picture matching tasks or tasks probing semantic knowledge about a concept are based on the retrieval of distinctive information (Devlin et al., 1998), the poor performance of $\mathrm{AD}$ patients on these tasks may reflect early damage to this kind of semantic information.

Better preservation of shared than distinctive semantic knowledge in our $\mathrm{AD}$ patients was documented by their performance on the semantic Questionnaire. Indeed, while AD 
patients' performance did not significantly differ from that of NCs on the superordinate questions, which tap a level of knowledge common to all concepts of a given category, demented patients scored significantly lower than NCs on the subordinate questions, which explore knowledge of attributes that allow distinguishing one concept from another in the same category. The dissociation found between performance accuracy on shared versus distinctive semantic attributes on the same intentional task (besides that found between automatic and intentional tasks) suggests that the performance of $\mathrm{AD}$ patients on a particular task is more predictable in terms of the kind of knowledge investigated than on the basis of intentional or automatic access to semantic information. The semantic priming paradigm used in the present study did not allow us to investigate whether integrity of semantic priming in $\mathrm{AD}$ patients is actually a function of the kind of semantic relationship linking the prime to the target. However, the results of two recent semantic priming studies with degenerative demented patients are in keeping with this view (Moss \& Tyler, 1999; Giffard et al., 2002).

The different distribution of shared and distinctive features among concept representations belonging to living and non-living domains has been considered at the base of the emergence of specific patterns of category deficits during the course of AD pathology. As previously mentioned, according to Devlin et al.'s model (Devlin et al., 1998), in the early stages of the disease, a relative disadvantage for non-living things should be expected because these representations are supported by more vulnerable, distinctive, non-correlated features. Only later, when a catastrophic loss of whole sets of shared and correlated attributes of concepts occurs, a reversed category effect, that is, poorer performance on living than on non-living items is expected. However, different predictions can be made on the basis of Moss et al.'s (1998) model. As in Devlin et al.'s model (Devlin et al., 1998), in this one correlated features are thought to be more resistant to damage because they are sustained by collateral activation. However, although Moss and Tyler also posit that non-living things are represented by more distinctive features than living concepts (represented more by shared and correlated features), in this case the distinctive properties of non-living things are supposed to be more strongly correlated (and, as a consequence, more resistant to damage) than the distinctive features of living things. Accordingly, in early stages of the disease, patients with a diffuse semantic memory impairment will be particularly poor on the living items of any test requiring preservation of distinctive knowledge. Only later, when damage involves the distinctive features of non-living things and, at the end, when the highly correlated shared features of both living and non-living domains are also involved, can a relative advantage for living things be detected.

Our finding of three AD patients with a prevalent impairment for the living category on the semantic battery and the absence of such a dissociation on the priming task contrasts with Gonnerman et al.'s hypothesis (Gonnerman et al., 1997) of an early prevalent deficit for non-living things. On the contrary, this result fits well with Moss and Tyler's prediction (Moss et al., 1998). Indeed, the three AD patients with a mild level of pathology may have been particularly poor on the living items of the semantic battery because performance on these tasks requires the integrity of distinctive information which, due to its poor correlation, is supposed to be most affected in this category. On the other side, if priming facilitation is based on the activation of patterns of units that overlap in different concepts, these patients should have normal priming facilitation in both living and non-living categories. This is due to the integrity of shared information in both semantic domains at this stage of the disease.

\subsection{Conclusions}

Results of the present study show that a disadvantage for living rather than non-living things can be observed even in normal subjects. Several factors are likely responsible for this "physiologic" asymmetry, such as general properties intrinsic to individual items and qualitative differences in the semantic representation of different conceptual categories. Failure to detect a category-specific semantic deficit in our sample of AD patients was probably due to the lack of a prevalent impairment for the living category in most of the patients studied. This finding is in keeping with previous research showing that category-specific effects may not be a generalised phenomenon in $\mathrm{AD}$ but may pertain to a limited number of patients (Garrard et al., 1998). Finally, we found that the category-specific effect could not be confirmed in a semantic priming paradigm in those $\mathrm{AD}$ patients with a specific impairment for living category on the intentional semantic tasks. Different hypothesis have been advanced to explain this result. However, recent findings make us lean more toward the interpretative framework based on the hypotheses maintaining that the variability of performance in an AD sample depends greatly on the portion of semantic knowledge explored by the experimental task. Although the present investigation provides only indirect support for it, further experimental work, directly investigating this issue is currently under way in our laboratory.

\section{References}

Barbarotto, R., Capitani, E., \& Laiacona, M. (1996). Naming deficit in herpes simplex encephalitis. Acta Neurologica Scandinavica, 93, 211216.

Battig, W. F., \& Montague, W. E. (1969). Category norms for verbal items in 56 categories: a replication and extension of the Connecticut category norms. Journal of Experimental Psychology Monography, 80, part 2.

Bayles, K. A., \& Tomoeda, C. K. (1983). Confrontational naming impairment in dementia. Brain and Language, 19, 98-114.

Bortolini, V., Tagliavini, C., \& Zampolli, A. (1971). Lessico di frequenze della lingua italiana contemporanea. Milano: Garzanti.

Capitani, E., Laiacona, M., \& Barbarotto, R. (1993). Dissociazioni semantiche intracategoriali. Pare II. Procedura automatica di analisi 
di una batteria standardizzata. Archivio di Psicologia, Neurologia e Psichiatria, 54, 457-476.

Capitani, E., Laiacona, M., Barbarotto, R., \& Trivelli, C. (1994). Living and non-living categories. Is there a "normal" asymmetry? Neuropsychologia, 32, 1453-1463.

Chenery, H. J., Ingram, J. C. L., \& Murdoch, B. (1994). The effect of repeated prime-target presentation in manipulating attention-induced priming in persons with dementia of the Alzheimer's type. Brain and Cognition, 25, 108-127.

Chertkow, H., \& Bub, D. (1989). Priming and semantic memory loss in Alzheimer's disease. Brain and Language, 36, 420-446.

Chertkow, H., Bub, D., Bergman, H., Bruemmer, A., Merling, A., \& Rothfleisch, J. (1994). Increased semantic priming in patients with dementia of the Alzheimer's type. Journal of Clinical and Experimental Neuropsychology, 16, 608-622.

Collins, A. M., \& Loftus, E. F. (1975). A spreading activation theory of semantic processing. Psychological Review, 82, 407-428.

Daum, I., Riesch, G., Sartori, G., \& Birbaumer, N. (1996). Semantic memory impairment in Alzheimer's disease. Journal of Clinical and Experimental Neuropsychology, 18, 648-665.

Devlin, J. T., Gonnerman, L. M., Andersen, E. S., \& Seidenberg, M. S. (1998). Category-specific semantic deficits in focal and widespread brain damage: a computational account. Journal of Cognitive Neuroscience, 10, 77-94.

Farah, M. J., \& McClelland, J. L. (1991). A computational model of semantic memory impairment: modality specificity and emergent category specificity. Journal of Experimental Psychology: General, 120, 339-357.

Funnell, E., \& Sheridan, J. S. (1992). Categories of knowledge? Unfamiliar aspects of living and non-living things. Cognitive Neuropsychology, 9, 135-153.

Gaffan, D., \& Heywood, C. A. (1993). A spurious category-specific visual agnosia for living things in normal human and non-human primates. Journal of Cognitive Neuroscience, 5, 118-128.

Gainotti, G. (1990). The categorical organization of semantic and lexical knowledge in the brain. Behavioural Neurology, 3, 109-115.

Gainotti, G., Di Betta, A. M., \& Silveri, M. C. (1996). The production of specific and generic associates of living and non-living. Brain and Language, 54, 262-274.

Garrard, P., Lambon-Ralph, M. A., Hodges, J. R., \& Patterson, K. (2001). Prototipicality, distinctivness, and intercorrelations: analyses of the semantic attributes of living and non-living concepts. Cognitive Neuropsychology, 18, 125-174.

Garrard, P., Patterson, K., Watson, P. C., \& Hodges, J. R. (1998). Category-specific semantic loss in dementia of Alzheimer's type. Functional-anatomical correlations from cross-sectional analyses. Brain, 121, 633-646.

Giffard, B., Desgranges, B., Nore-Mary, F., Lavelèe, C., Beaunieux, H., de la Sayette, V., Pasquier, F., \& Eustache, F. (2002). The dynamic course of semantic memory impairment in Alzheimer's disease: clues from hyperpriming and hypopriming effects. Brain, 125, 2044-2057.

Gonnerman, L. M., Andersen, E. S., Devlin, J. T., Kempler, D., \& Seidenberg, M. S. (1997). Double dissociation of semantic categories in Alzheimer's disease. Brain and Language, 57, 254-279.

Hair, J. F., Anderson, R. E., Tatham, R. L., \& Balch, W. C. (1998). Multiple discriminant analysis and logistic regression. In Multivariate data analysis (5th ed., p. 282). Englewood Cliffs, NJ: Prentice-Hall.

Hartman, M. (1991). The use of semantic knowledge in Alzheimer's disease: evidence for impairments of attention. Neuropsychologia, 29, 213-228.

Hodges, J. R., Salmon, D. P., \& Butters, N. (1992). Semantic memory impairment in Alzheimer's disease: failure of access or degraded knowledge? Neuropsychologia, 30, 301-314.

Kerlinger, F. N., \& Pedhazur, E. J. (1973). In Rinehart, \& Winston (Eds.), Multiple regression in behavioral research. New York: Holt.

Laiacona, M., Barbarotto, R., Trivelli, C., \& Capitani, E. (1993). Perceptual and associative knowledge in category-specific impairment of semantic memory: a study of two cases. Cortex, 29, 727-740.
Laiacona, M., Barbarotto, R., Trivelli, C., \& Capitani, E. (1993). Dissociazioni semantiche intercategoriali: descrizione di una batteria standardizzata e dati normativi. Archivio di Psicologia, Neurologia e Psichiatria, 54, 209-248.

Lambon Ralph, M. A., Howard, D., Nightingale, G., \& Ellis, A. W. (1998). Are living and non-living category-specific deficits causally linked to impaired perceptual or associative knowledge? Evidence from a category-specific double dissociation. Neurocase, 4, 311-338.

Martin, A., \& Fedio, P. (1983). Word production and comprehension in Alzheimer's disease: the breakdown of semantic knowledge. Brain and Language, 19, 124-141.

Masson, M. E. J. (1995). A distributed memory model of semantic priming. Journal of Experimental Psychology: Learning, Memory and Cognition, 21, 3-23.

Mazzoni, M., Moretti, P., Lucchini, C., Vista, M., \& Muratorio, A. (1991). Category-specific semantic disorders in Alzheimer's disease. Rivista di Neurologia, 1, 77-85.

McClelland, J. L., \& Rumelhart, D. E. (1981). An interactive activation model of context effects in letter perception. An account of basic findings. Psychological Review, 88, 357-407.

McKhann, G., Drachman, D., Folstein, M., Katzman, R., Price, D., \& Stadlan, E. (1984). Clinical diagnosis of Alzheimer's disease: report of the NINCDS-ADRDA Work Group under the auspices of the Department of Health and Human Service Task Force in Alzheimer's disease. Neurology, 34, 939-944.

McRae, K., De Sa, V. R., \& Seidenberg, M. S. (1997). On the nature and scope of featural representation of word meaning. Journal of Experimental Psychology: General, 126, 99-130.

Montanes, P., Goldblum, M. C., \& Boller, F. (1995). Naming colour and black-white pictures from living and non-living things in Alzheimer's disease. Journal of the International Neuropsychological Society, 1, $39-48$.

Montanes, P., Goldblum, M. C., \& Boller, F. (1996). Classification deficits in Alzheimer's disease with special reference to living and non-living things. Brain and Language, 54, 335-358.

Morrison, C. M., Chappell, T. D., \& Ellis, A. W. (1997). Age of acquisition norms for a large set of object names and their relation to adult estimates and other variables. Quarterly Journal of Experimental Psychology, 50, 528-529.

Moss, H. E., \& Tyler, L. K. (1999). A progressive category-specific semantic deficit for non-living things. Neuropsychologia, 38, 60-82.

Moss, H. E., Tyler, L. K., Durrant-Peatfield, M., \& Bunn, E. M. (1998). Two eyes of a see-through: impaired and intact semantic knowledge in a case of selective deficit for living things. Neurocase, 4, 291-310.

Nebes, R. D., Brady, C. B., \& Huff, F. (1989). Automatic and attentional mechanisms of semantic priming in Alzheimer's disease. Journal of Clinical and Experimental Neuropsychology, 11, 219-230.

Nebes, R. D., Martin, D., \& Horn, L. C. (1984). Sparing of semantic memory in Alzheimer's disease. Journal of Abnormal Psychology, 93, 321-330.

Neely, J. H. (1977). Semantic priming and retrieval from lexical memory: roles of inhibitionless spreading activation and limited-capacity attention. Journal of Experimental Psychology: General, 106, 226-254.

Neely, J. H. (1991). Semantic priming effects in visual word recognition: a selective review of current findings and theories. In D. Besner, \& G. W. Humphreys (Eds.), Basic processes in reading: Visual word recognition (pp. 264-336). Hillsdale, NJ: Erlbaum.

Ober, B. A., Shenaut, G. K., Jagust, W. J., \& Syillman, R. C. (1991). Automatic semantic priming with various category relations in Alzheimer's disease and normal aging. Psychology and Aging, 6, 647-660.

Parasuraman, R., \& Martin, A. (1994). Cognition in Alzheimer's disease: disorders of attention and semantic knowledge. Current Opinion in Neurobiology, 4, 237-244.

Rapp, B., \& Caramazza, A. (1993). On the distinction between deficits of access and deficits of storage: a question of theory. Cognitive Neuropsychology, 10, 113-141. 
Rumelhart, D. E., \& McClelland, J. L. (1986). Parallel distributed models (Vol. 1). Cambridge, MA; MIT Press.

Salmon, D. P., Butters, N., \& Chan, A. S. (1999). The deterioration of semantic memory in Alzheimer's disease. Canadian Journal of Experimental Psychology, 53, 108-117.

Shallice, T. (1988). From neuropsychology to mental structure. Cambridge: Cambridge University Press.

Silveri, M. C., Daniele, A., Giustolisi, L., \& Gainotti, G. (1991). Dissociation between knowledge of living and non-living things in dementia of the Alzheimer type. Neurology, 41, 545-546.

Silveri, M. C., Monteleone, D., Burani, C., \& Tabossi, P. (1996). Automatic semantic facilitation in Alzheimer's disease. Journal of Clinical and Experimental Neuropsychology, 18, 371-382.
Snodgrass, J. G., \& Vanderwart, M. A. (1980). A standardized set of 260 pictures: norms for name agreement, image agreement, familiarity and visual complexity. Journal of Experimental Psychology: Human Learning and Memory, 6, 174-215.

Tippett, L. J., Grossman, M., \& Farah, M. J. (1996). The semantic memory impairment of Alzheimer's disease: category-specific? Cortex, $32,143-153$.

Warrington, E. K., \& Shallice, T. (1984). Category-specific semantic impairments. Brain, 107, 829-854.

Zannino, G. D., Perri, R., Carlesimo, G. A., \& Caltagirone, C. (2002), Category-specific impairment in patients with Alzheimer's disease as a function of disease severity: a cross-sectional investigation. Neurpsychologia, 40, 2268-2279. 